\title{
Total Synthesis of the Reported Structure of Cahuitamycin A
}

\author{
Justin A. Shapiro ${ }^{1}$, Savannah J. Post ${ }^{1}$, William M. Wuest ${ }^{1,2 *}$ \\ ${ }^{1}$ Department of Chemistry, Emory University, Atlanta, GA, 30322, United States \\ ${ }^{2}$ Emory Antibiotic Resistance Center, Emory University, Atlanta, GA 30322, United States.
}

Keywords: Total Synthesis, Natural Products, Antibiotics, Structure Elucidation, Biofilm.

\begin{abstract}
In a 2016 screen of natural product extracts a new family of natural products, the cahuitamycins, was discovered and found to inhibit the formation of biofilms in the human pathogen Acinetobacter baumannii. The molecules contain an unusual piperazate residue that raises structure/function and biosynthesis questions and resemble iron-trafficking virulence factors from $A$. baumannii, suggesting a connection between metal homeostasis and biofilm-mediated pathogenicity. Here we disclose the first total synthesis of the reported structure of cahuitamycin A in a twelve-step longest linear sequence and $18 \%$ overall yield. Comparison of spectral data of the authentic natural product and synthetic target compound demonstrate that the reported structure is distinct from the isolated metabolite. Herein, we propose an alternative structure to reconcile our findings with the isolation report, setting the stage for future synthetic and biochemical investigations of an important class of natural products.
\end{abstract}

Multi-drug resistant bacterial infections represent a global health crisis, as outlined by the Centers for Disease Control and Prevention threat report on antibiotic resistance. ${ }^{1}$ Particularly troubling are resistant Acinetobacter baumannii, which were upgraded from "serious threat" in the 2013 report $^{2}$ to "urgent threat" in 2019 due to the ease with which resistance determinants can spread amongst this species and the lack of antibiotics in the clinic or pipeline to treat infections. A. baumannii is an opportunistic pathogen commonly found in pneumonia, wound, bloodstream, and urinary tract infections amongst immunecompromised patients and those in hospitals or military treatment facilities. Isolates are routinely found with resistance to drugs of last resort such as the carbapenems and polymyxins. ${ }^{3}$ With effective treatment options dwindling, new drugs with novel mechanisms that are less likely to succumb to resistance are desperately needed to combat this increasing threat.

An important method by which A. baumannii evades treatment is by the formation of biofilms, ${ }^{4}$ communities of bacteria bound together by extracellular matrices which display enhanced tolerance to antibiotics (10-1000X) and are known to lay dormant on medical equipment. In a natural product extract library screen followed by extensive ribosomal engineering, Sherman and coworkers identified a novel group of natural products from Streptomyces gandocaensis called the cahuitamycins ${ }^{5}$ (Figure 1A). These compounds were shown to

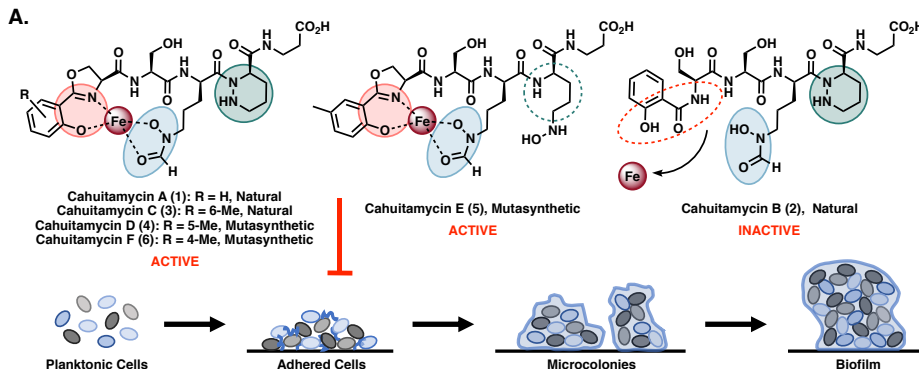

Figure 1. A) Structures \& bioactivity of the cahuitamycins; B) Siderophores of A. baumannii; C) Piperazate-containing natural products. selectively inhibit the formation of $A$. baumannii biofilm formation with minimal effects on planktonic growth. Structural characterization identified the cahuitamycins as highly functionalized non-ribosomal hexapeptides which originate from the same biosynthetic gene cluster. The parent compound, cahuitamycin A (1), is proposed to originate from salicylic acid, two units of serine, two units of ornithine, and $\beta$-alanine. Among the interesting structural features in this molecule are the $o$-phenolate oxazoline and the terminal hydroxamate, both strong iron-chelating moieties found in A. baumannii siderophores $^{6-8}$ (Figure 1B), and the piperazate, a rare amino acid residue found in highly bioactive and structurally diverse natural products such as the kutznerides ${ }^{9}$ and the sanglifehrins ${ }^{10}$ (Figure 1C). Uncyclized shunt product cahuitamycin B (2) lacks the phenolate oxazoline and has no bioactivity. By recognizing that cahuitamycin C (3) is derived from an off-cluster starting material, the authors were able to engineer mutants deficient in starter-unit biosynthesis to generate mutasynthetic analogs via feeding experiments, resulting in cahuitamycins D (4), $\mathrm{F}^{11}(6)$, and $\mathrm{E}(\mathbf{5})$, which lacks the piperazate moiety.

The isolation of cahuitamycin E illustrates ambiguity surrounding the biosynthetic origins of the piperazate in the natural product scaffold. This can be rationalized either by on-enzyme cyclization or non-specific loading of multiple substrates onto the domain. To date, only KtzT from the kutzneride pathway B.

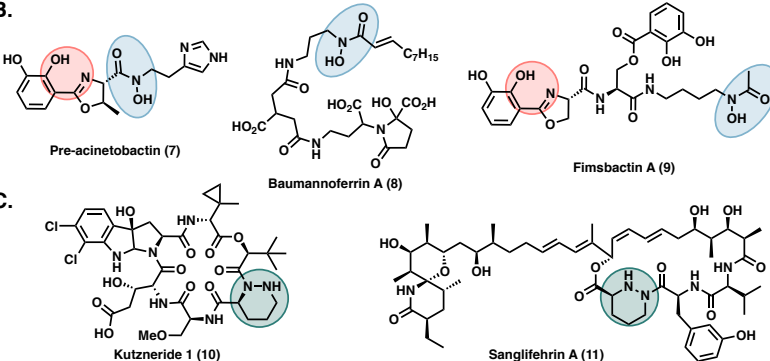


Scheme 1. Synthesis of key intermediate (+)-21.<smiles>CCNCCCC(CC(C)(C)C)C(=O)O</smiles>

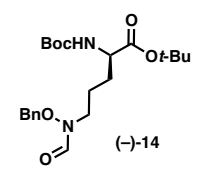

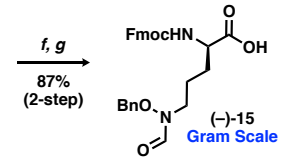
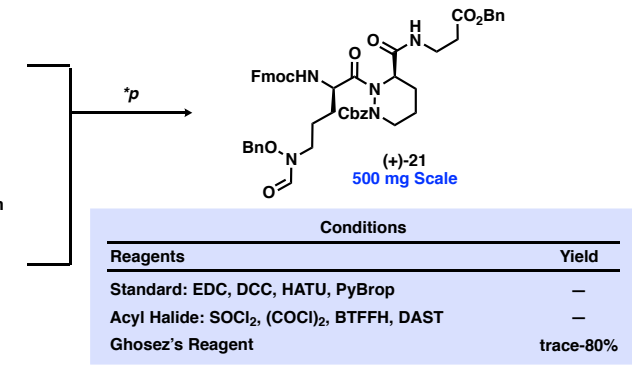

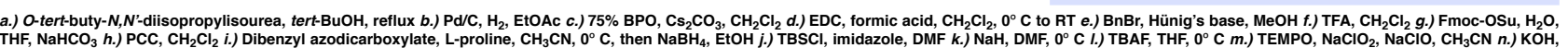
THF o.) EDC, HOBt, DMF, NEt 3 , $\beta$-alanine benzyl ester TsOH, $0^{\circ} \mathrm{C}$ to RT p. $\left.^{*}\right)^{*}$ Optimized Conditions: (-)-15, Ghosez's Reagent, $0^{\circ} \mathrm{C}$, then (+)-20, AgCN, benzene, reflux. See Supporting Table 4 for full reaction screen.

has been functionally characterized and demonstrated to catalyze the key N-N bond formation to generate monomeric piperazic acid, ${ }^{12}$ implying that cahuitamycin $\mathrm{E}$ is not a shunt product but a result of combinatorial biosynthesis. This apparent flexibility, along with incorporation of methyl-substituted salicylates into the key pharmacophore, demonstrates that the cahuitamycins are a dynamic system ripe for investigation.

These results naturally invite a complementary synthetic approach to answer key questions regarding the structure and activity of the cahuitamycins. Given the unique incorporation of orthogonal bioactive moieties and their potent activity against a critical virulence behavior in highly relevant human pathogens, we embarked on the diverted total synthesis of cahuitamycin A and simplified analogs to provide a framework for future study of this important natural product.

Our diverted total synthetic approach relied on the convergent coupling of three peptides - wherein the linchpin ornithine fragment ${ }^{13}$ would join the piperazate- $\beta$-alanine and phenolate oxazoline moieties. Toward this end, the central ornithine fragment was furnished from $N_{\alpha}$-Boc- $N_{\varepsilon}$-Cbz-D-ornithine (-)-12, which was first converted to a tert-butyl ester via an isourea reagent quantitatively (Scheme 1, top). After Cbz deprotection, the terminal N-O bond was formed by reaction with $75 \%$ benzoyl peroxide and cesium carbonate to afford (-)-13 in $75 \%$ yield over three steps. Formylation with EDC followed by a one-pot protecting-group exchange gave (-)-14 in $85 \%$ yield over two steps. The Boc and tert-butyl groups were then

Scheme 2. Endgame synthesis of $(+)-1$ and proline analog $(+)-26$ (see SI for full details).
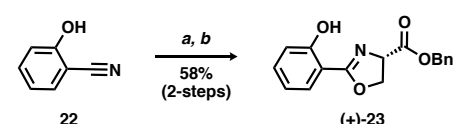

$(+)-23$
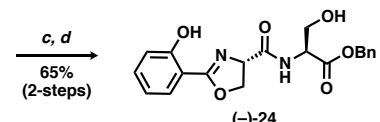

$250 \stackrel{(-)-24}{\mathrm{mg} \text { Scale }}$
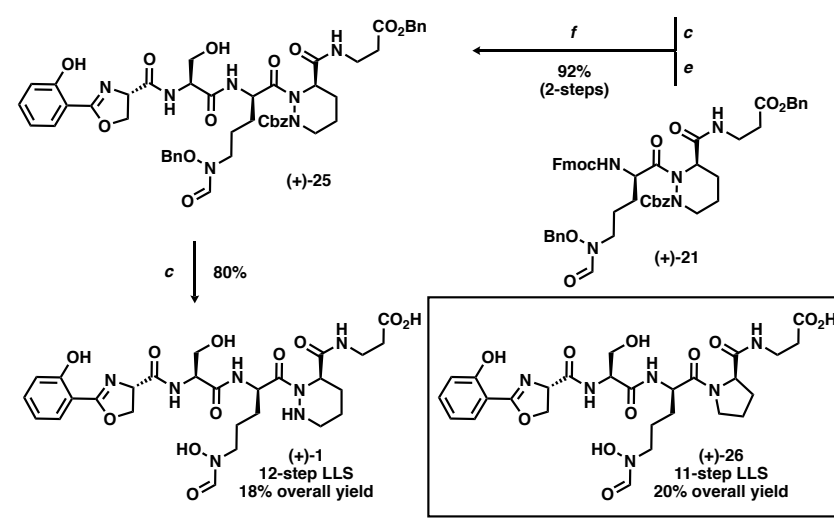

Conditions: a.) AcCl, $\mathrm{MeOH}$ b.) 1,2-dichloroethane, L-serine benzyl ester $\mathrm{HCl}$, reflux c.) $\mathrm{Pd} / \mathrm{C}, \mathrm{H}_{2}, \mathrm{MeOH}$ d.) EDC, $\mathrm{HOBt}, \mathrm{DMF}, \mathrm{NEt}_{3}$, L-serine benzyl ester $\mathrm{HCl}, 0^{\circ} \mathrm{C}$ to $\mathrm{RT}$ e.) 4 -(aminomethyl)piperidine, $\mathrm{CH}_{2} \mathrm{Cl}_{2}$ then $\mathrm{pH} 5.5$ buffer $f$.) $\mathrm{EDC}, \mathrm{HOBt}, \mathrm{CH}_{3} \mathrm{CN}, \mathrm{NEt}_{3}, 0^{\circ} \mathrm{C}$ to $\mathrm{RT}$ removed simultaneously by TFA and the amine was re-protected with Fmoc-OSu, giving the linchpin fragment (-)-15 in $87 \%$ yield over two steps on gram scale.

In parallel, oxidation of alcohol $\mathbf{1 6}$ to the corresponding aldehyde, followed by stereoselective installation of the hydrazine mediated via in situ iminium catalysis and subsequent reduction gave (-)-17 in 67\% yield over two steps. After TBS-protection, the linear hydrazine was cyclized into piperazic silyl ether $(+)$ 18 in $81 \%$ yield over two steps. The silyl group was removed with TBAF quantitatively and then oxidized to afford the di$\mathrm{Cbz}$ protected carboxylic acid, ${ }^{14}$ which was regioselectively deprotected by stirring in pulverized potassium hydroxide ${ }^{15}$ to afford (+)-19 in 75\% yield over three steps. Coupling with $\beta$ alanine benzyl ester resulted in $(+)-20$ in $77 \%$ yield on gram scale (Scheme 1, bottom).

As expected, the coupling between acid (-)-15 and piperazate (+)-20 was non-trivial. ${ }^{16}$ After standard coupling reagents failed, we turned to the literature for procedures known to mediate couplings between non-nucleophilic amines and hindered acids. Ultimately, we found that pre-stirring acid (-)-15 in Ghosez's reagent ${ }^{17}$ followed by reflux in benzene with silver cyanide resulted in tripeptide $(+)-21$ in reasonable yields. By altering equivalents of acid and piperazate, the product was obtained reliably in $80 \%$ isolated yield (Scheme 1, right).

Finally, synthesis of the heterocyclic moiety began from commercial nitrile 22. Oxazoline $(+)-23$ was achieved through the corresponding imidate and cyclization with $\mathrm{L}-\mathrm{Ser}-\mathrm{CO}_{2} \mathrm{Bn}$ in refluxing 1,2-DCE (Scheme 2, top). After hydrogenolysis, EDC/HOBt coupling gave fragment (-)-24. Reductive deprotection of (-)-24 was performed in parallel to Fmoc-cleavage of (+)-21 by 4-AMP and the two deprotected materials were subjected to EDC coupling conditions furnishing $(+)-25$ in modest yield. Judicious solvent selection and further reaction optimization increased the yield to $92 \%$ over two steps. Global deprotection provided the final product $(+)-1$ in $80 \%$ yield. In total, the target structure was achieved in a longest linear sequence of 12 steps and $18 \%$ overall yield.

In a parallel manner, we also set out to synthesize the simplified proline analog (+)-26 (Scheme 2) which is bioisosteric with the piperazate moiety ${ }^{18}$ and more readily available commercially. We posited that this analog could serve as a simplified chemical probe that would be easily accessible via our diverted total synthetic route. Toward this end, we synthesized the analog in an 11-step longest linear sequence, in $20 \%$ overall yield. Full synthetic details are provided in the supporting information.

With both synthetic samples and authentic material (provided by Sherman) in hand, we sought to fully characterize the natural product and confirm its structure. ${ }^{1} \mathrm{H}$ NMR of our synthetic 
product $(+)-1$ showed substantial differences from the authentic isolated material. Signals from the salicylate, oxazoline, serine, and $\beta$-alanine moieties matched well between natural productand synthetic (+)-1. However, signals associated with the piperazate and the ornithine residues shifted by up to $\sim 1.0$ ppm (Table S1). By contrast, ${ }^{1} \mathrm{H}-\mathrm{NMR}$ signals from proline analog (+)-26 mapped well onto authentic material (Figure 2).

Taken together, we considered two possible explanations that would account for these discrepancies - (1) stereochemical misassignment or (2) alternative structural connectivity of the piperazate moiety. We note that the isolationists presented extensive and compelling data for structural assignment including two-dimensional NMR, advanced Marfey's analysis, and bioinformatic examination of the biosynthetic gene cluster. Any alternate proposed structure would need to reconcile our findings with these data. We took note that while in silico analysis of the NRPS assembly line predicted the substrate for most of the modules, there was ambiguity surrounding the incorporation of the piperazic acid. Accordingly, the stereochemical mis-assignment of cahuitamycin A would contradict the Marfey's analysis conducted by the Sherman group and would also not explain the relative similarity between the ${ }^{1} \mathrm{H}-\mathrm{NMR}$ signals for the authentic sample and proline analog $(+)-26$.

A second, and more likely, solution to this structural question would be the incorrect assignment of bond connectivity within the natural product. We propose an alternative connectivity of cahuitamycin A (27), a structural isomer of the proposed molecule, bonded between the distal nitrogen of the piperazate (akin to that of sanglifehrin) and the D-ornithine residue. Importantly, this structure is consistent with the 2D-NMR (HMBC correlation from position 5-to-9 and from position 8-to-9) and Marfey's analysis (the hydrolyzed amino acid monomers from compounds (+)-1 and 27 would be the same) presented in the isolation report. We are presently investigating this hypothesis and the results will be published in due course.
We further characterized our two synthetic compounds and the natural product by testing inhibition against growth of $\mathrm{Aci}$ netobacter baumannii ATCC 17978 (Figure 3). As expected, authentic cahuitamycin A inhibited growth to comparable levels as reported in the isolation paper $\left(\mathrm{IC}_{50}\right.$ of $\left.\sim 18 \mu \mathrm{M}\right)$. By contrast, synthetic $(+)-1$ and proline analog $(+)-26$ showed no growth inhibition and in fact provided a growth advantage, perhaps due to their structural similarity to known $A$. baumannii siderophores and an ability to act as surrogate iron-sources. These results also provide evidence that while proline is known to serve as a bioisosteric substitute for piperazates when connected at N2, this activity likely does not hold for the structural isomer.

The biosynthetic origins of piperazate-containing natural products is a young area of study ${ }^{19}$ and our findings have important implications for biochemists studying the incorporation of these unusual amino acids into secondary metabolites. Our proposed scaffold would be only the second class of natural products bound at $\mathrm{N} 1$ of the piperazate (the first being the sanglifehrins) and the only class to be synthesized entirely on an NRPS assembly line. Structural elucidation of such molecules is made challenging by ambiguous bioinformatics and limitations of traditional spectroscopic techniques. In light of our results, it is possible that similar reevaluation of other reported piperazate natural products is warranted. This work illustrates the value of total synthesis as a complementary approach to traditional biochemical techniques in understanding the activity and biosynthetic logic of complex natural products.

These results also impact organic chemists studying antibiotic natural products, especially those targeting bacterial virulence. Siderophore-mediated iron-acquisition as an antibiotic target has been a topic of broad interest ${ }^{20}$ and has recently even resulted in an FDA approved drug (cefiderocol, brand name Fetroja $\left.{ }^{\circledR}\right){ }^{21}$ The SAR from the isolation report points to an asof-yet unidentified relationship between the activity of the

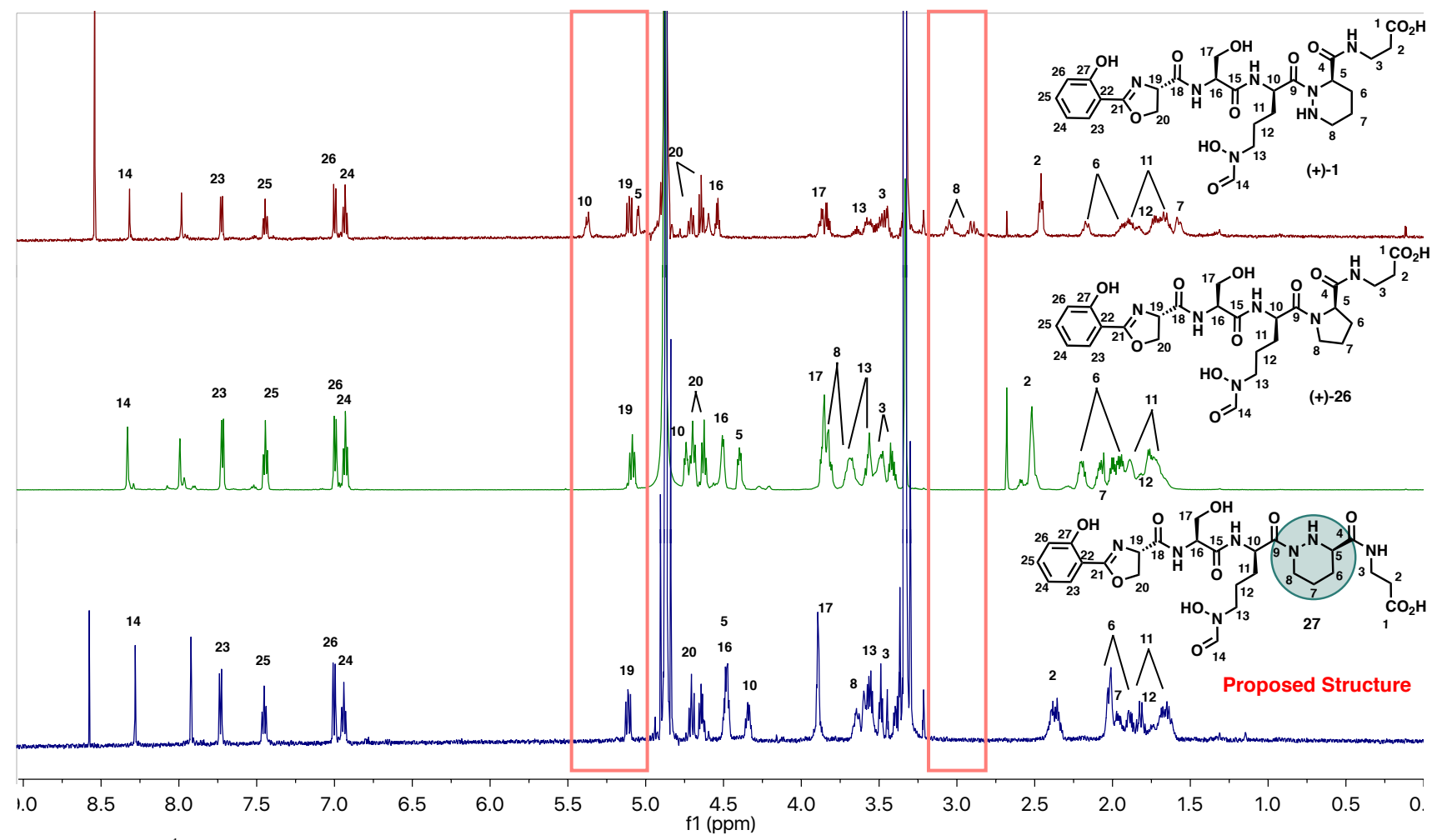

Figure 2. ${ }^{1} \mathrm{H}-\mathrm{NMR}$ spectra of synthetic compound (+)-1 (top), proline analog $(+)-26$ (middle), and authentic cahuitamycin A (bottom). 
Growth Inhibition Against $A$. baumannii

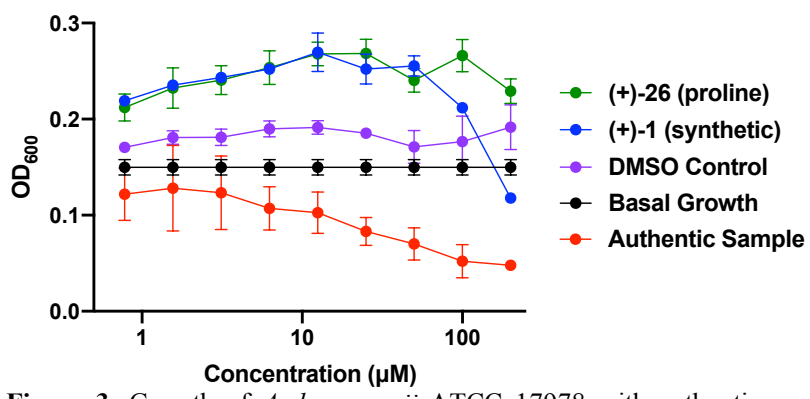

Figure 3. Growth of $A$. baumannii ATCC 17978 with authentic cahuitamycin A (red), synthetic (+)-1 (blue), $(+)-26$ (green), and DMSO (purple). Error bars represent SD from three independent replicates.

cahuitamycins and their similarity to the siderophores of $\mathrm{A}$. baumannii. This indicates either (1) that the cahuitamycins are Trojan-horse antibiotics which enter the target cell through siderophore uptake machinery and then inhibit by an unrelated mechanism, or (2) the cahuitamycins inhibit A. baumannii by directly interfering with siderophore cycling. Given that a subtle structural change between (+)-1 and authentic natural product is enough to abolish activity, one infers a fairly specific interaction with some target protein as opposed to a general mechanism. These results invite further investigation into the cahuitamycins as tool compounds to probe the interplay between bacterial virulence traits.

For decades, biochemistry and organic chemistry have worked in tandem to identify novel compounds from nature with unique bioactivity and improve upon their structures in the search for novel molecular mechanisms and targets. In this work, we have executed a concise and efficient diverted total synthesis of the reported structure of cahuitamycin A and a simplified analog. By careful analysis of the spectral data and biological activity of these compounds in comparison with the authentic material, we provide insight into the true structure of the natural product. This sets the stage for future synthetic efforts to definitively answer a molecular mystery posed by the microbial world. This work, yet again, highlights the power of synthetic organic chemistry to better understand and utilize the tools from nature's chemical inventory.

\section{ASSOCIATED CONTENT}

\section{AUTHOR INFORMATION}

\section{Corresponding Author}

*wwuest@emory.edu

\section{Author Contributions}

All authors contributed to the experimental design, writing, and approve of the final version of the manuscript.

\section{ACKNOWLEDGMENT}

We are grateful to the NIH (GM119426 to W.M.W.; F32 GM133091 to J.A.S.) and NSF (DGE1937971 to S.J.P) for financial support. We are indebted to Prof. David Sherman and Dr. Ashootosh Tripathi for thoughtful discussion and providing the authentic material for comparison.

\section{REFERENCES}

(1) Centers for Disease Control and Prevention. Antibiotic Resistance Threats in the United States. 2019.

$$
\text { Chen, Y.; Lu, Y.; Zou, Q.; Chen, H.; Ma, D. A Scalable Process }
$$
Asymmetry 2004, 15, 3477-3481. to the Key Intermediate of Cilazapril, (S)-1Benzyloxycarbonylhexahydropyridazine-3-Carboxylic Acid, Through a Novel Cascade Course. Org. Process. Res. Dev. 2013, 17, 1209-1213.

(16) Oelke, A. J.; France, D. J.; Hofmann, T.; Wuitschik, G.; Ley, S. V. Piperazic Acid-Containing Natural Products: Isolation, Biological Relevance and Total Synthesis. Nat. Prod. Rep. 2011, $28,1445-1471$.

(17) Devos, A.; Remion, J.; Frisque-Hesbain, A. M.; Colens, A.; Ghosez, L. Synthesis of Acyl Halides under Very Mild Conditions. J. Chem. Soc. Chem. Commun. 1979, 24, 1180-1181. Hu, Y.; Qi, Y.; Stumpf, S. D.; D’Alessandro, J. M.; Blodgett, J. A. V. Bioinformatic and Functional Evaluation of Actinobacterial Piperazate Metabolism. ACS Chem. Biol. 2019, 14, 696-703.

Morgan, K. D.; Andersen, R. J.; Ryan, K. S. Piperazic AcidContaining Natural Products: Structures and Biosynthesis. Nat. Prod. Rep. 2019, 36, 1628-1653.

Gilda Tonziello, E. C.; Biagio Pinchera, G. G.; Petrosillo, N. Present and Future of Siderophore-Based Therapeutic and Diagnostic Approaches in Infectious Diseases. Infect. Dis. Rep. 2019, 11, 8208 .

Wu, J. Y.; Srinivas, P.; Pogue, J. M. Cefiderocol: A Novel Agent for the Management of Multidrug-Resistant Gram-Negative Organisms. Infect. Dis. Ther. 2020, 9, 17-40. 
Table of Contents Image:
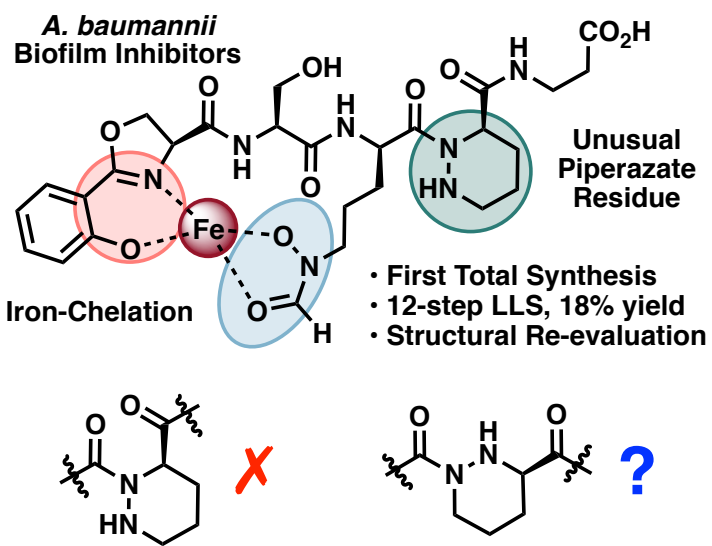

? 